\title{
Analysis of reaction-diffusion systems where a parameter influences both the reaction terms as well as the boundary
}

\author{
A. Acharya ${ }^{1}$, N. Fonseka ${ }^{2}$ and R. Shivaji ${ }^{1 *}$
}

${ }^{\text {*Correspondence: shivaji@uncg.edu }}$ ${ }^{1}$ Department of Mathematics and Statistics, University of North Carolina at Greensboro, Greensboro, NC 27412, USA

Full list of author information is available at the end of the article

\section{Abstract}

We study positive solutions to steady-state reaction-diffusion models of the form

$$
\left\{\begin{array}{l}
-\Delta u=\lambda f(v) ; \quad \Omega, \\
-\Delta v=\lambda g(u) ; \quad \Omega, \\
\frac{\partial u}{\partial \eta}+\sqrt{\lambda} u=0 ; \quad \partial \Omega, \\
\frac{\partial v}{\partial \eta}+\sqrt{\lambda} v=0 ; \quad \partial \Omega,
\end{array}\right.
$$

where $\lambda>0$ is a positive parameter, $\Omega$ is a bounded domain in $\mathbb{R}^{N}(N>1)$ with smooth boundary $\partial \Omega$, or $\Omega=(0,1), \frac{\partial z}{\partial \eta}$ is the outward normal derivative of $z$. We assume that $f$ and $g$ are continuous increasing functions such that $f(0)=0=g(0)$ and $\lim _{s \rightarrow \infty} \frac{f(M g(s))}{s}=0$ for all $M>0$. In particular, we extend the results for the single equation case discussed in (Fonseka et al. in J. Math. Anal. Appl. 476(2):480-494, 2019) to the above system.

MSC: $35 J 15 ; 35 J 25 ; 35 J 60$

\section{Introduction}

In [7] the authors analyzed and established several results for positive solutions for reaction-diffusion models of the form

$$
\left\{\begin{array}{l}
-\Delta u=\lambda f(u) ; \quad \Omega, \\
\frac{\partial u}{\partial \eta}+\mu(\lambda) u=0 ; \quad \partial \Omega,
\end{array}\right.
$$

where $f \in C^{2}([0, \infty))$, and $\mu \in C([0, \infty))$ is strictly increasing such that $\mu(0) \geq 0$. In recent history, there has been a lot of interests in models where the parameter influences the equation and boundary conditions (see [5-7], and [8]). In this paper, we are interested in

(c) The Author(s) 2021. This article is licensed under a Creative Commons Attribution 4.0 International License, which permits use, sharing, adaptation, distribution and reproduction in any medium or format, as long as you give appropriate credit to the original author(s) and the source, provide a link to the Creative Commons licence, and indicate if changes were made. The images or other third party material in this article are included in the article's Creative Commons licence, unless indicated otherwise in a credit line to the material. If material is not included in the article's Creative Commons licence and your intended use is not permitted by statutory regulation or exceeds the permitted use, you will need to obtain permission directly from the copyright holder. To view a copy of this licence, visit http://creativecommons.org/licenses/by/4.0/. 
extending the results in [7] to systems of the form

$$
\begin{cases}-\Delta u=\lambda f(v) ; & \Omega, \\ -\Delta v=\lambda g(u) ; & \Omega, \\ \frac{\partial u}{\partial \eta}+\sqrt{\lambda} u=0 ; & \partial \Omega, \\ \frac{\partial v}{\partial \eta}+\sqrt{\lambda} v=0 ; & \partial \Omega\end{cases}
$$

where $\lambda>0$ is a positive parameter, $\Omega$ is a bounded domain in $\mathbb{R}^{N}(N>1)$ with smooth boundary $\partial \Omega$, or $\Omega=(0,1), \frac{\partial z}{\partial \eta}$ is the outward normal derivative of $z$, and $f, g$ satisfy the following conditions:

$\left(H_{1}\right) f, g \in C[0, \infty)$, increasing, $f(0)=0=g(0)$, and $\lim _{s \rightarrow \infty} \frac{f(M g(s))}{s}=0$ for all $M>0$ (combined sublinear effect at infinity). Further, there exists $a>0$ such that $f, g \in C^{1}[0, a)$ and $f^{\prime}(0), g^{\prime}(0)>0$.

Without loss of generality, we assume that $f^{\prime}(0) \geq g^{\prime}(0)$ throughout the paper. We first recall recent results for the eigenvalue problem

$$
\left\{\begin{array}{l}
-\Delta v=E v ; \quad \Omega, \\
\frac{\partial v}{\partial \eta}+\gamma \sqrt{E} v=0 ; \quad \partial \Omega,
\end{array}\right.
$$

where $\gamma>0$. Namely, let $E_{1}(\gamma)$ be its principal eigenvalue (see [8]), and let $v$ be the corresponding normalized positive eigenfunction of (1.2). Now consider the eigenvalue problem

$$
\begin{cases}-\Delta \phi=\bar{E} g^{\prime}(0) \phi ; & \Omega, \\ \frac{\partial \phi}{\partial \eta}+\sqrt{\bar{E}} \phi=0 ; & \partial \Omega .\end{cases}
$$

Noting that the substitution $E=\bar{E} g^{\prime}(0)$ reduces (1.3) to (1.2), we easily see that the principal eigenvalue of (1.3) is $\frac{E_{1}(\gamma)}{g^{\prime}(0)}$ with $\gamma=\frac{1}{\sqrt{g^{\prime}(0)}}$. Define

$$
A_{1}:=\frac{E_{1}(\gamma)}{g^{\prime}(0)}
$$

Next, for a given $\tilde{\lambda}$, we recall the for the eigenvalue problem

$$
\begin{cases}-\Delta v=(\sigma+\tilde{\lambda}) v ; & \Omega, \\ \frac{\partial v}{\partial \eta}+\gamma \sqrt{\tilde{\lambda}} v=0 ; & \partial \Omega .\end{cases}
$$

Denoting by $\sigma_{\tilde{\lambda}}(\tilde{\lambda}, \gamma)$ its principal eigenvalue and by $\phi_{\tilde{\lambda}}>0$ the corresponding eigenfunction of (1.5) such that $\left\|\phi_{\tilde{\lambda}}\right\|=1$, the following results were established in [8]:

$$
\begin{cases}\sigma_{\tilde{\lambda}}>0 ; & \tilde{\lambda}<E_{1}(\gamma), \\ \sigma_{\tilde{\lambda}}=0 ; & \tilde{\lambda}=E_{1}(\gamma), \\ \sigma_{\tilde{\lambda}}<0 ; & \tilde{\lambda}>E_{1}(\gamma) .\end{cases}
$$


Hence, by the substitution $\lambda g^{\prime}(0)=\tilde{\lambda}$, denoting by $\sigma_{\lambda}$ the principal eigenvalue of

$$
\left\{\begin{array}{l}
-\Delta \phi=\left(\sigma+\lambda g^{\prime}(0)\right) \phi ; \quad \Omega \\
\frac{\partial \phi}{\partial \eta}+\sqrt{\lambda} \phi=0 ; \quad \partial \Omega
\end{array}\right.
$$

we deduce the following:

$$
\begin{cases}\sigma_{\lambda}>0 ; & \lambda<A_{1}, \\ \sigma_{\lambda}=0 ; & \lambda=A_{1}, \\ \sigma_{\lambda}<0 ; & \lambda>A_{1} .\end{cases}
$$

Next, for $0<a<b$, define

$$
Q_{1}(a):=\min \left\{\frac{a}{f(a)}, \frac{a}{g(a)}\right\}
$$

and

$$
Q_{2}(b):=\max \left\{\frac{b}{f(b)}, \frac{b}{g(b)}\right\}
$$

Further, let

$$
C_{1}=\inf _{\epsilon} \frac{N}{\epsilon^{N}} \frac{R^{N-1}}{R-\epsilon}
$$

where $R$ is the radius of the largest inscribed ball in $\Omega$. Let $w$ be the unique solution of

$$
\left\{\begin{array}{l}
-\Delta w=1 ; \quad \Omega \\
\frac{\partial w}{\partial \eta}+w=0 ; \quad \partial \Omega
\end{array}\right.
$$

Then we first establish the following:

Theorem 1.1 Let $\left(H_{1}\right)$ hold. Then (1.1) has a positive solution $\left(u_{\lambda}, v_{\lambda}\right)$ for $\lambda>A_{1}$ such that $\left\|u_{\lambda}\right\|_{\infty},\left\|v_{\lambda}\right\|_{\infty} \rightarrow \infty$ as $\lambda \rightarrow \infty$. Further, if there exists $0<a<b$ such that $\frac{Q_{1}}{Q_{2}}>C_{1}\|w\|_{\infty}$ and $Q_{1}>\max \left\{A_{1}, 1\right\}\|w\|_{\infty}$, then (1.1) has at least three positive solutions for

$$
\max \left\{A_{1}, C_{1} Q_{2}, 1\right\}<\lambda<\frac{Q_{1}}{\|w\|_{\infty}} \quad \text { (see Fig. 1). }
$$

Next we establish the following:

Theorem 1.2 If there exists $r>0$ such that $f, g \in C^{2}([0, r)), f(0)=0=g(0), f^{\prime}(0)=g^{\prime}(0)>0$, and $f^{\prime \prime}(s), g^{\prime \prime}(s)<0$ for all $s \in[0, r)$, then (1.1) has a positive solution $\left(u_{\lambda}, v_{\lambda}\right)$ for $\lambda>A_{1}$ and $\lambda \approx A_{1}$ such that $\left\|u_{\lambda}\right\|_{\infty},\left\|v_{\lambda}\right\|_{\infty} \rightarrow 0$ as $\lambda \rightarrow A_{1}^{+}$(see Fig. 2). 
Figure 1 Bifurcation diagram for positive solutions when the hypothesis of Theorem 1.1 holds

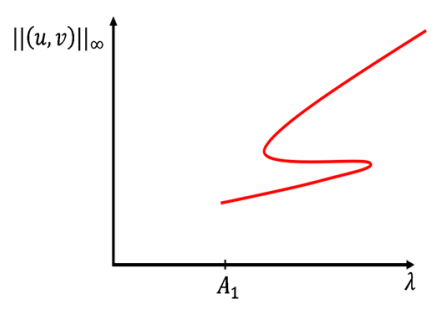

Figure 2 Bifurcation diagram for positive solutions when the hypotheses of Theorems 1.1 and 1.2 hold

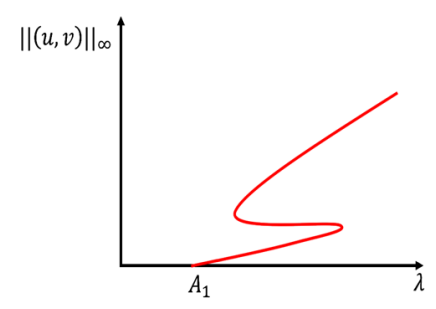

Remark 1.1 Note that if both $\frac{s}{f(s)}$ and $\frac{s}{g(s)}$ are strictly increasing for $s>0$, then the multiplicity of positive solutions for $\lambda>A_{1}$ is not possible (see Appendix).

We present some preliminaries in Sect. 2. We provide proofs of Theorems 1.1 and 1.2 in Sect. 3. In Sect. 4, we discuss an example. We state and prove Lemma 5.1 in Appendix to justify Remark 1.1. Our existence and multiplicity results are established via the method of sub- and supersolutions. We conclude this Introduction by citing two additional related references [3] and [9].

\section{Preliminaries}

In this section, we introduce definitions of a subsolution and a supersolution of (1.1), and state sub-supersolution theorems which are used to prove the existence and multiplicity for positive solutions. By a subsolution of (1.1) we mean $\left(\psi_{1}, \psi_{2}\right) \in C^{2}(\Omega) \cap C^{1}(\bar{\Omega})$ that satisfies

$$
\begin{cases}-\Delta \psi_{1} \leq \lambda f\left(\psi_{2}\right) ; & \Omega, \\ -\Delta \psi_{2} \leq \lambda g\left(\psi_{1}\right) ; & \Omega, \\ \frac{\partial \psi_{1}}{\partial \eta}+\sqrt{\lambda} \psi_{1} \leq 0 ; & \partial \Omega, \\ \frac{\partial \psi_{2}}{\partial \eta}+\sqrt{\lambda} \psi_{2} \leq 0 ; & \partial \Omega,\end{cases}
$$

and by a supersolution of $(1.1)$ we mean $\left(Z_{1}, Z_{2}\right) \in C^{2}(\Omega) \cap C^{1}(\bar{\Omega})$ that satisfies

$$
\begin{cases}-\Delta Z_{1} \geq \lambda f\left(Z_{2}\right) ; & \Omega, \\ -\Delta Z_{2} \geq \lambda g\left(Z_{1}\right) ; & \Omega, \\ \frac{\partial Z_{1}}{\partial \eta}+\sqrt{\lambda} Z_{1} \geq 0 ; & \partial \Omega, \\ \frac{\partial Z_{2}}{\partial \eta}+\sqrt{\lambda} Z_{2} \geq 0 ; & \partial \Omega .\end{cases}
$$

Now we state two results (see $[2,10]$, and $[11])$ we will use later. 
Lemma 2.1 Let $\left(\psi_{1}, \psi_{2}\right)$ and $\left(Z_{1}, Z_{2}\right)$ be a subsolution and a supersolution of $(1.1)$, respectively, such that $\left(\psi_{1}, \psi_{2}\right) \leq\left(Z_{1}, Z_{2}\right)$. Then (1.1) has a solution $(u, v) \in C^{2}(\Omega) \cap C^{1}(\bar{\Omega})$ such that $(u, v) \in\left[\left(\psi_{1}, \psi_{2}\right),\left(Z_{1}, Z_{2}\right)\right]$.

Lemma 2.2 Let $f$ and $g$ be nonnegative and increasing, and suppose there exist a subsolution $\left(\psi_{1}, \bar{\psi}_{1}\right)$, a strict supersolution $\left(\phi_{2}, \bar{\phi}_{2}\right)$, a strict subsolution $\left(\psi_{2}, \bar{\psi}_{2}\right)$, and a supersolution $\left(\phi_{1}, \bar{\phi}_{1}\right)$ for $(1.1)$ such that $\left(\psi_{1}, \bar{\psi}_{1}\right) \leq\left(\psi_{2}, \bar{\psi}_{2}\right) \leq\left(\phi_{1}, \bar{\phi}_{1}\right),\left(\psi_{1}, \bar{\psi}_{1}\right) \leq\left(\phi_{2}, \bar{\phi}_{2}\right) \leq$ $\left(\phi_{1}, \bar{\phi}_{1}\right)$, and $\left(\psi_{2}, \bar{\psi}_{2}\right) \not\left(\phi_{2}, \bar{\phi}_{2}\right)$. Then (1.1) has at least three positive solutions $\left(u_{i}, v_{i}\right), i=$ $1,2,3$, such that $\left(u_{1}, v_{1}\right) \in\left[\left(\psi_{1}, \bar{\psi}_{1}\right),\left(\phi_{2}, \bar{\phi}_{2}\right)\right],\left(u_{2}, v_{2}\right) \in\left[\left(\psi_{2}, \bar{\psi}_{2}\right),\left(\phi_{1}, \bar{\phi}_{1}\right)\right]$, and $\left(u_{3}, v_{3}\right) \in$ $\left[\left(\psi_{1}, \bar{\psi}_{1}\right),\left(\phi_{1}, \bar{\phi}_{1}\right)\right] \backslash\left(\left[\left(\psi_{1}, \bar{\psi}_{1}\right),\left(\phi_{2}, \bar{\phi}_{2}\right)\right] \cup\left[\left(\psi_{2}, \bar{\psi}_{2}\right),\left(\phi_{1}, \bar{\phi}_{1}\right)\right]\right)$.

\section{Proofs of Theorems 1.1 and 1.2}

Proof of Theorem 1.1 First, we show the existence of positive solutions for $\lambda>A_{1}$. Let $\left(\psi_{1}, \psi_{2}\right)=\left(m \phi_{\lambda}, m \phi_{\lambda}\right)$, where $\phi_{\lambda}$ is the normalized positive eigenfunction of (1.7). Define $H(s)=\left(\sigma_{\lambda}+\lambda g^{\prime}(0)\right) s-\lambda f(s)$. Then $H(0)=0, H^{\prime}(0)=\sigma_{\lambda}+\lambda\left(g^{\prime}(0)-f^{\prime}(0)\right)<0$ (by (1.8)). This implies that $H(s) \leq 0$ for $s \approx 0$. Thus $\left(\sigma_{\lambda}+\lambda g^{\prime}(0)\right) m \phi_{\lambda}-\lambda f\left(m \phi_{\lambda}\right) \leq 0$ for $m \approx 0$. Hence $-\Delta \psi_{1} \leq \lambda f\left(\psi_{2}\right)$ for $m \approx 0$. Analyzing $\tilde{H}(s)=\left(\sigma_{\lambda}+\lambda g^{\prime}(0)\right) s-\lambda g(s)$, by a similar argument we obtain $-\Delta \psi_{2} \leq \lambda g\left(\psi_{1}\right)$. Further, on the boundary, we have $\frac{\partial \psi_{1}}{\partial \eta}+\sqrt{\lambda} \psi_{1}=0$ and $\frac{\partial \psi_{2}}{\partial \eta}+$ $\sqrt{\lambda} \psi_{2}=0$. Thus $\left(\psi_{1}, \psi_{2}\right)$ is a subsolution of (1.1) for $m \approx 0$.

Now let $e_{\lambda}$ be a positive solution of

$$
\left\{\begin{array}{l}
-\Delta e=1 ; \quad \Omega, \\
\frac{\partial e}{\partial \eta}+\sqrt{\lambda} e=0 ; \quad \partial \Omega .
\end{array}\right.
$$

We consider three different cases. If both $f$ and $g$ are bounded, then let $\left(Z_{1}, Z_{2}\right)=$ $\left(\lambda M_{\lambda} \frac{e_{\lambda}}{\left\|e_{\lambda}\right\|_{\infty}}, \lambda M_{\lambda} \frac{e_{\lambda}}{\left\|e_{\lambda}\right\|_{\infty}}\right)$ and choose $M_{\lambda}$ large such that $\frac{M_{\lambda} \lambda}{\left\|e_{\lambda}\right\|_{\infty}} \geq \lambda f\left(\frac{M_{\lambda} e_{\lambda}}{\left\|e_{\lambda}\right\|_{\infty}}\right)$. This implies $-\Delta Z_{1}-\lambda f\left(Z_{2}\right) \geq 0$ for $M_{\lambda} \gg 1$, and by a similar argument we see that $-\Delta Z_{2}-\lambda g\left(Z_{1}\right) \geq 0$ for $M_{\lambda} \gg 1$. Also, on the boundary, we have $\frac{\partial Z_{1}}{\partial \eta}+\sqrt{\lambda} Z_{1}=0$ and $\frac{\partial Z_{2}}{\partial \eta}+\sqrt{\lambda} Z_{2}=0$. Hence $\left(Z_{1}, Z_{2}\right)$ is a supersolution for $M_{\lambda} \gg 1$. If $g(x) \rightarrow \infty$ as $x \rightarrow \infty$, let $\left(Z_{1}, Z_{2}\right)=$ $\left(M_{\lambda} e_{\lambda}, \lambda g\left(M_{\lambda}\left\|e_{\lambda}\right\|_{\infty}\right) e_{\lambda}\right)$. Then by choosing $M_{\lambda}$ large we obtain

$$
\frac{1}{\lambda\left\|e_{\lambda}\right\|_{\infty}} \geq \frac{f\left(\lambda\left\|e_{\lambda}\right\|_{\infty} g\left(M_{\lambda}\left\|e_{\lambda}\right\|_{\infty}\right)\right)}{M_{\lambda}\left\|e_{\lambda}\right\|_{\infty}}
$$

which implies that $M_{\lambda}-\lambda f\left(\lambda g\left(M_{\lambda}\left\|e_{\lambda}\right\|_{\infty}\right) e_{\lambda}\right) \geq 0$. Hence $-\Delta Z_{1}-\lambda f\left(Z_{2}\right) \geq 0$. We also have $\lambda g\left(M_{\lambda}\left\|e_{\lambda}\right\|_{\infty}\right)-\lambda g\left(M_{\lambda} e_{\lambda}\right) \geq 0$. This implies that $-\Delta Z_{2}-\lambda g\left(Z_{1}\right) \geq 0$. Further, on the boundary, we have $\frac{\partial Z_{1}}{\partial \eta}+\sqrt{\lambda} Z_{1}=\frac{\partial Z_{2}}{\partial \eta}+\sqrt{\lambda} Z_{2}=0$. Hence $\left(Z_{1}, Z_{2}\right)$ is a supersolution of (1.1) for $M_{\lambda} \gg 1$. Finally, we consider the case where $f(x) \rightarrow \infty$ as $x \rightarrow \infty$ and $g(x)$ is bounded. In this case, let $\left(Z_{1}, Z_{2}\right)=\left(\lambda f\left(M_{\lambda}\left\|e_{\lambda}\right\|_{\infty}\right) e_{\lambda}, M_{\lambda} e_{\lambda}\right)$. Then $\lambda f\left(M_{\lambda}\left\|e_{\lambda}\right\|_{\infty}\right)-\lambda f\left(M_{\lambda} e_{\lambda}\right) \geq 0$, which implies that $-\Delta Z_{1}-\lambda f\left(Z_{2}\right) \geq 0$. Also, we have $M_{\lambda} \geq \lambda g\left(\lambda f\left(M_{\lambda}\left\|e_{\lambda}\right\|_{\infty}\right) e_{\lambda}\right)$ for $M_{\lambda} \gg 1$. This implies that $-\Delta Z_{2}-\lambda g\left(Z_{1}\right) \geq 0$. Further, on the boundary, we have $\frac{\partial Z_{1}}{\partial \eta}+\sqrt{\lambda} Z_{1}=$ $\frac{\partial Z_{2}}{\partial \eta}+\sqrt{\lambda} Z_{2}=0$. Hence $\left(Z_{1}, Z_{2}\right)$ is a supersolution of (1.1) for $M_{\lambda} \gg 1$. Now we can choose $M_{\lambda}$ large enough such that $\left(\psi_{1}, \psi_{2}\right) \leq\left(Z_{1}, Z_{2}\right)$. Hence by Lemma (2.1), (1.1) has a positive solution for $\lambda>A_{1}$.

Next, we show that there exists a positive solution $\left(u_{\lambda}, v_{\lambda}\right)$ for $\lambda \gg 1$ such that $\left\|u_{\lambda}\right\|_{\infty},\left\|v_{\lambda}\right\|_{\infty} \rightarrow \infty$ as $\lambda \rightarrow \infty$. Define $h \in C^{2}([0, \infty))$ such that $h(0)<0, h(s) \leq f(s)$, and 
$h(s) \leq g(s)$ for $s \in(0, \infty)$ and $\lim _{s \rightarrow \infty} h(s)>0$. Then the Dirichlet boundary value problem

$$
\left\{\begin{array}{l}
-\Delta w=\lambda h(w) ; \quad \Omega, \\
w=0 ; \quad \partial \Omega,
\end{array}\right.
$$

has a positive solution $\bar{w}_{\lambda}$ for $\lambda \gg 1$ such that $\left\|\bar{w}_{\lambda}\right\|_{\infty} \rightarrow \infty$ as $\lambda \rightarrow \infty$ (see [4]).

It is easy to show that $\left(\bar{w}_{\lambda}, \bar{w}_{\lambda}\right)$ is a subsolution of (1.1) for $\lambda \gg 1$ since $h \leq f, h \leq g$, and $\frac{\partial \bar{w}}{\partial \eta}<0 ; \partial \Omega$. We can also choose $M_{\lambda} \gg 1$ such that $\left(Z_{1}, Z_{2}\right) \geq\left(\bar{w}_{\lambda}, \bar{w}_{\lambda}\right)$, where $\left(Z_{1}, Z_{2}\right)$ is a supersolution of (1.1) as constructed before. By Lemma 2.1, (1.1) has a positive solution $\left(u_{\lambda}, v_{\lambda}\right) \in\left[\left(\bar{w}_{\lambda}, \bar{w}_{\lambda}\right),\left(Z_{1}, Z_{2}\right)\right]$ for $\lambda \gg 1$. Clearly, $\left\|u_{\lambda}\right\|_{\infty},\left\|v_{\lambda}\right\|_{\infty} \rightarrow \infty$ as $\lambda \rightarrow \infty$ since $\|\bar{w}\|_{\infty} \rightarrow \infty$ as $\lambda \rightarrow \infty$.

Next, we establish our multiplicity result. We first construct a positive strict supersolution $\left(\tilde{z}_{1}, \tilde{z}_{2}\right)$ for $(1.1)$ when $1<\lambda<\frac{Q_{1}}{\|w\|_{\infty}}$, where $w$ is the solution of (1.12). Let $\left(\tilde{z}_{1}, \tilde{z}_{2}\right)=$ $\left(a \frac{w}{\|w\|_{\infty}}, a \frac{w}{\|w\|_{\infty}}\right)$. Then $\frac{a}{\|w\|_{\infty} f(a)}>\lambda$ gives us $\frac{a}{\|w\|_{\infty}}>\lambda f(a)$. Since $f$ is increasing, we have $\frac{a}{\|w\|_{\infty}} \geq \lambda f\left(a \frac{w}{\|w\|_{\infty}}\right)$, which implies that $-\Delta \tilde{z}_{1} \geq \lambda f\left(\tilde{z}_{2}\right)$ in $\Omega$. Similarly, we can show that $-\Delta \tilde{z}_{2} \geq \lambda g\left(\tilde{z}_{1}\right)$ in $\Omega$. On the boundary, we have $\frac{\partial \tilde{z}_{1}}{\partial \eta}+\sqrt{\lambda} \tilde{z}_{1}=\frac{a}{\|w\|_{\infty}}\left[\frac{\partial w}{\partial \eta}+\sqrt{\lambda} w\right]>\frac{a}{\|w\|_{\infty}}\left[\frac{\partial w}{\partial \eta}+\right.$ $w]=0$ since $\lambda>1$. Similarly, we have $\frac{\partial \tilde{z}_{2}}{\partial \eta}+\sqrt{\lambda} \tilde{z}_{2}>0$. Thus $\left(\tilde{z}_{1}, \tilde{z}_{2}\right)$ is a strict supersolution of (1.1).

Now we construct a strict subsolution $\left(\tilde{\psi}_{1}, \tilde{\psi}_{2}\right)$ of $(1.1)$ for $\lambda \geq C_{1} Q_{2}=C_{1} \max \left\{\frac{b}{g(b)}, \frac{b}{f(b)}\right\}$. Note that in [1] the authors showed that the boundary value problem

$$
\left\{\begin{array}{l}
-\Delta u=\lambda f(v) ; \quad \Omega, \\
-\Delta v=\lambda g(u) ; \quad \Omega, \\
u=0 ; \quad \partial \Omega, \\
v=0 ; \quad \partial \Omega,
\end{array}\right.
$$

has a strict subsolution $\left(\bar{u}_{0}, \bar{v}_{0}\right)$ for $\lambda \geq C_{1} \max \left\{\frac{b}{g(b)}, \frac{b}{f(b)}\right\}$ such that $\left\|\bar{u}_{0}\right\|_{\infty} \geq b$ and $\left\|\bar{v}_{0}\right\|_{\infty} \geq$ $b$. Let $\left(\tilde{\psi}_{1}, \tilde{\psi}_{2}\right)$ be the first iteration of $\left(\bar{u}_{0}, \bar{v}_{0}\right)$, that is, the solution to the problem

$$
\begin{cases}-\Delta \tilde{\psi}_{1}=\lambda f\left(\bar{v}_{0}\right) ; & \Omega, \\ -\Delta \tilde{\psi}_{2}=\lambda g\left(\bar{u}_{0}\right) ; & \Omega, \\ \frac{\partial \tilde{\psi}_{1}}{\partial \eta}+\sqrt{\lambda} \tilde{\psi}_{1}=0 ; & \partial \Omega, \\ \frac{\partial \tilde{\psi}_{2}}{\partial \eta}+\sqrt{\lambda} \tilde{\psi}_{2}=0 ; & \partial \Omega .\end{cases}
$$

Then by comparison principle $\left(\tilde{\psi}_{1}, \tilde{\psi}_{2}\right)>\left(\bar{u}_{0}, \bar{v}_{0}\right) ; \bar{\Omega}$. Hence $\left(\tilde{\psi}_{1}, \tilde{\psi}_{2}\right)$ is a strict subsolution of (1.1) such that $\left\|\tilde{\psi}_{1}\right\|_{\infty} \geq b>a$ and $\left\|\tilde{\psi}_{2}\right\|_{\infty} \geq b>a$. Thus we have $\left(\tilde{\psi}_{1}, \tilde{\psi}_{2}\right) \not \leq\left(\tilde{z}_{1}, \tilde{z}_{2}\right)$. Further, for $\lambda>A_{1}$, we can construct the subsolution $\left(\psi_{1}, \psi_{2}\right)$ as before for $m \approx 0$, and for any $\lambda>0$, we can construct the supersolution $\left(Z_{1}, Z_{2}\right)$ as before for $M_{\lambda} \gg 1$. Also, for $m \approx 0$ and $M_{\lambda} \gg 1$, we obtain $\left(\psi_{1}, \psi_{2}\right) \leq\left(\tilde{\psi}_{1}, \tilde{\psi}_{2}\right) \leq\left(Z_{1}, Z_{2}\right)$ and $\left(\psi_{1}, \psi_{2}\right) \leq$ $\left(\tilde{z}_{1}, \tilde{z}_{2}\right) \leq\left(Z_{1}, Z_{2}\right)$. Hence by Lemma (2.2), (1.1) has at least three positive solutions for $\lambda \in\left(\max \left\{A_{1}, C_{1} Q_{2}, 1\right\}, \frac{Q_{1}}{\|w\|_{\infty}}\right)$.

Proof of Theorem 1.2 Let $\left(\psi_{1}, \psi_{2}\right)=\left(m \phi_{\lambda}, m \phi_{\lambda}\right)$ be as in the proof of Theorem 1.1. Then $\left(\psi_{1}, \psi_{2}\right)$ is a subsolution of $(1.1)$ for $m \approx 0$. Since $f^{\prime \prime}(s)<0$ and $g^{\prime \prime}(s)<0$ for $s \approx 0$, there 
exists $A>0$ such that $f^{\prime \prime}(s) \leq-A$ and $g^{\prime \prime}(s) \leq-A$ for $s \approx 0$. Let $\left(\phi_{1}, \phi_{2}\right)=\left(\delta_{\lambda} \phi_{\lambda}, \delta_{\lambda} \phi_{\lambda}\right)$, where $\delta_{\lambda}=-\frac{2 \sigma_{\lambda}}{\lambda A \min \bar{\Omega}_{\lambda}}$. Note that $\delta_{\lambda}>0$ and $\delta_{\lambda} \rightarrow 0$ as $\lambda \rightarrow A_{1}^{+}$since $\sigma_{\lambda} \rightarrow 0$ and $\min _{\bar{\Omega}} \phi_{\lambda} \nrightarrow 0$ as $\lambda \rightarrow A_{1}^{+}$(see [8]). Clearly, $\left\|\phi_{1}\right\|_{\infty} \rightarrow 0$ and $\left\|\phi_{2}\right\|_{\infty} \rightarrow 0$ as $\lambda \rightarrow A_{1}^{+}$. Then by Taylor's theorem we have $f\left(\phi_{2}\right)=f(0)+f^{\prime}(0) \phi_{2}+\frac{f^{\prime \prime}(\xi)}{2} \phi_{2}^{2}=\phi_{2}+\frac{f^{\prime \prime}(\xi)}{2} \phi_{2}^{2}$ for some $\xi \in\left[0, \phi_{2}\right]$. Then we have

$$
\begin{aligned}
-\Delta \phi_{1}-\lambda f\left(\phi_{2}\right) & =\delta_{\lambda}\left(\sigma_{\lambda}+\lambda g^{\prime}(0)\right) \phi_{\lambda}-\lambda\left[\delta_{\lambda} \phi_{\lambda} f^{\prime}(0)+\frac{f^{\prime \prime}(\xi)}{2}\left(\delta_{\lambda} \phi_{\lambda}\right)^{2}\right] \\
& \geq \delta_{\lambda} \phi_{\lambda}\left[\sigma_{\lambda}+\frac{\lambda A}{2} \delta_{\lambda} \min _{\bar{\Omega}} \phi_{\lambda}\right]=0
\end{aligned}
$$

by our choice of $\delta_{\lambda}$. A similar argument shows that $-\Delta \phi_{2}-\lambda g\left(\phi_{1}\right) \geq 0$. Further, on the boundary, we have $\frac{\partial \phi_{1}}{\partial \eta}+\sqrt{\lambda} \phi_{1}=\frac{\partial \phi_{2}}{\partial \eta}+\sqrt{\lambda} \phi_{2}=0$. Thus $\left(\phi_{1}, \phi_{2}\right)$ is a supersolution of (1.1). Choosing $m \approx 0$, we also have $\left(\psi_{1}, \psi_{2}\right) \leq\left(\phi_{1}, \phi_{2}\right)$. By Lemma 2.1 there exists a positive solution $\left(u_{\lambda}, v_{\lambda}\right) \in\left[\left(\psi_{1}, \psi_{2}\right),\left(\phi_{1}, \phi_{2}\right)\right]$ for $\lambda>A_{1}$ and $\lambda \approx A_{1}$ such that $\left\|u_{\lambda}\right\|_{\infty} \rightarrow 0,\left\|v_{\lambda}\right\|_{\infty} \rightarrow$ 0 as $\lambda \rightarrow A_{1}^{+}$.

\section{Example}

For an example to illustrate Theorems 1.1-1.2, consider $f=f_{\alpha, k}$ and $g=g_{k}$ as follows:

$$
\begin{aligned}
& f=f_{\alpha, k}(s)= \begin{cases}e^{\frac{s}{s+1}}-1 ; & s \leq k, \\
{\left[e^{\frac{\alpha s}{\alpha+s}}-e^{\frac{\alpha k}{\alpha+k}}\right]+\left[e^{\frac{k}{k+1}}-1\right] ;} & s \geq k,\end{cases} \\
& g=g_{k}(s)= \begin{cases}2(1+s)^{\frac{1}{2}}-2 ; & s \leq k, \\
{\left[\frac{1}{2}(1+s)^{2}-\frac{1}{2}(1+k)^{2}\right]+\left[2(1+k)^{\frac{1}{2}}-2\right] ;} & s \geq k,\end{cases}
\end{aligned}
$$

where $k>0$ is a constant, and $\alpha>0$ is a parameter. Note that though $g$ is superlinear at infinity, since $f$ is bounded, $\frac{f(M g(s))}{s} \longrightarrow 0$ as $s \longrightarrow \infty$ for all $M>0$. Choose $a=k, b=\alpha$, and $\alpha>k$. Then we note that $Q_{1}(k)=\min \left\{\frac{k}{f(k)}, \frac{k}{g(k)}\right\} \longrightarrow \infty$ as $k \longrightarrow \infty$ since $\frac{k}{f(k)}, \frac{k}{g(k)} \longrightarrow \infty$ as $k \longrightarrow \infty$ and $Q_{2}(\alpha)=\max \left\{\frac{\alpha}{f(\alpha)}, \frac{\alpha}{g(\alpha)}\right\} \longrightarrow 0$ as $\alpha \longrightarrow \infty$ since $\frac{\alpha}{f(\alpha)}, \frac{\alpha}{g(\alpha)} \longrightarrow 0$ as $\alpha \longrightarrow$ $\infty$. Hence we can choose $k=k_{0}$ such that $Q_{1}\left(k_{0}\right)>\max \left\{A_{1}, 1\right\}\|w\|_{\infty}$ and choose $\alpha \gg 1$ such that $\frac{Q_{1}\left(k_{0}\right)}{Q_{2}(\alpha)} \geq C_{1}\|w\|_{\infty}$. Hence $\frac{Q_{1}}{\|w\|_{\infty}}>\max \left\{A_{1}, C_{1} Q_{2}, 1\right\}$ for $k=k_{0}$ and $\alpha \gg 1$. It is also clear that $f$ and $g$ satisfy $\left(H_{1}\right)$. This implies that (1.1) has at least one positive solution for $\lambda>A_{1}$ and at least three positive solutions for $\lambda \in\left(\max \left\{A_{1}, C_{1} Q_{2}, 1\right\}, \frac{Q_{1}}{\|w\|_{\infty}}\right)$ for $k=k_{0}$ and $\alpha \gg 1$. Thus Theorem 1.1 holds in this example for $k=k_{0}$ and $\alpha \gg 1$. Further, since $f^{\prime}(0)=g^{\prime}(0)$ and $f, g \in C^{2}([0, k))$ and $f^{\prime \prime}<0, g^{\prime \prime}<0$ for all $s \in[0, k)$, Theorem 1.2 holds. Hence the bifurcation diagram for positive solutions is as shown in Fig. 2.

\section{Appendix}

Lemma 5.1 Let $\left(H_{1}\right)$ hold, and let $\frac{s}{f(s)}$ and $\frac{s}{g(s)}$ be strictly increasing for $s>0$. Then (1.1) has a unique positive solution for $\lambda>A_{1}$.

Proof: The existence for $\lambda>A_{1}$ is clear from Theorem 1.1. Let $\left(u_{i}, v_{i}\right), i=1,2$, be two distinct positive solutions for $\lambda>A_{1}$. Since $\left(m \phi_{\lambda}, m \phi_{\lambda}\right)$ is a subsolution for $m \approx 0$, there exists a minimal positive solution, and hence without loss of generality, we can assume 
that $\left(u_{1}, v_{1}\right) \geq\left(u_{2}, v_{2}\right)$, by which we mean that $u_{1} \geq u_{2}, v_{1} \geq v_{2}$. Now

$$
\begin{aligned}
L & =\int_{\Omega}\left\{g\left(u_{1}\right) g\left(u_{2}\right)\left[\frac{u_{1}}{g\left(u_{1}\right)}-\frac{u_{2}}{g\left(u_{2}\right)}\right]+f\left(v_{1}\right) f\left(v_{2}\right)\left[\frac{v_{1}}{f\left(v_{1}\right)}-\frac{v_{2}}{f\left(v_{2}\right)}\right]\right\} d x \\
& =\int_{\Omega}\left[g\left(u_{2}\right) u_{1}-g\left(u_{1}\right) u_{2}+f\left(v_{2}\right) v_{1}-f\left(v_{1}\right) v_{2}\right] d x \\
& =\int_{\Omega}\left\{\left[u_{2} \Delta v_{1}-u_{1} \Delta v_{2}\right]+\left[v_{2} \Delta u_{1}-v_{1} \Delta u_{2}\right]\right\} d x \\
& =\int_{\partial \Omega}\left\{\left[u_{2} \frac{\partial v_{1}}{\partial \eta}-u_{1} \frac{\partial v_{2}}{\partial \eta}\right]+\left[v_{2} \frac{\partial u_{1}}{\partial \eta}-v_{1} \frac{\partial u_{2}}{\partial \eta}\right]\right\} d x \\
& =\int_{\partial \Omega}\left[-\sqrt{\lambda} u_{2} v_{1}+\sqrt{\lambda} u_{1} v_{2}-\sqrt{\lambda} v_{2} u_{1}+\sqrt{\lambda} v_{1} u_{2}\right] d x=0 .
\end{aligned}
$$

This is clearly a contradiction when $\frac{s}{f(s)}$ and $\frac{s}{g(s)}$ are strictly increasing and $\left(u_{1}, v_{1}\right),\left(u_{2}, v_{2}\right)$ are distinct. Thus (1.1) has a unique positive solution for $\lambda>A_{1}$.

\section{Acknowledgements}

None.

\section{Funding}

None.

Availability of data and materials

Not applicable.

\section{Competing interests}

The authors declare that they have no competing interests.

\section{Authors' contributions}

Equal contributions by all authors. All authors read and approved the final manuscript.

\section{Author details}

'Department of Mathematics and Statistics, University of North Carolina at Greensboro, Greensboro, NC 27412, USA.

${ }^{2}$ School of Arts and Sciences, Carolina University, Winston-Salem, NC 27101, USA.

\section{Publisher's Note}

Springer Nature remains neutral with regard to jurisdictional claims in published maps and institutional affiliations.

Received: 14 July 2020 Accepted: 18 January 2021 Published online: 01 February 2021

\section{References}

1. Ali, J., Ramaswamy, M., Shivaji, R.: Multiple positive solutions for classes of elliptic systems with combined nonlinear effects. Differ. Integral Equ. 19(6), 669-680 (2006)

2. Amann, H.: Fixed point equations and nonlinear eigenvalue problems in ordered Banach spaces. SIAM Rev. 18, 620-709 (1976)

3. Amster, P.: Multiple solutions for an elliptic system with indefinite Robin boundary conditions. Adv. Nonlinear Anal. 8(1), 603-614 (2019)

4. Castro, A., Garner, J.B., Shivaji, R.: Existence results for classes of sublinear semipositone problems. Results Math. 23, 214-220 (1993)

5. Cronin, J., Goddard, J., Shivaji, R.: Effects of patch-matrix composition and individual movement response on population persistence at the patch-level. Bull. Math. Biol. 81(10), 3933-3975 (2019)

6. Fonseka, N., Machado, J., Shivaji, R.: A study of logistic growth models influenced by the exterior matrix hostility and Grazing in an interior patch. Electron. J. Qual. Theory Differ. Equ. 17, 1-11 (2020)

7. Fonseka, N., Shivaji, R., Son, B., Spetzer, K.: Classes of reaction diffusion equations where a parameter influences the equation as well as the boundary condition. J. Math. Anal. Appl. 476(2), 480-494 (2019)

8. Goddard II, J., Morris, Q., Robinson, S., Shivaji, R.: An exact bifurcation diagram for a reaction diffusion equation arising in population dynamics. Bound. Value Probl. 2018, 170 (2018)

9. Hsu, C.H., Wu, S.L.: Periodic traveling fronts for partially degenerate reaction-diffusion systems with bistable and time-periodic nonlinearity. Adv. Nonlinear Anal. 9(1), 923-957 (2020)

10. Inkmann, F.: Existence and multiplicity theorems for semilinear elliptic equations with nonlinear boundary conditions. Indiana Univ. Math. J. 31, 213-221 (1982)

11. Shivaji, R.: A remark on the existence of three solutions via sub-super solutions. In: Lakshmikantham, V. (ed.) Nonlinear Analysis and Applications. Lecture Notes in Pure and Applied Mathematics, vol. 109, pp. 561-566 (1987) 